\title{
¿NO TENGO (AÚN O YA) LA EDAD? \\ (IN)VISIBILIDAD, VULNERABILIDAD Y VIOLENCIA DE LAS PERSONAS MENORES Y ANCIANAS
}

\author{
AM I NOT (YET OR ALREADY) OLD ENOUGH? \\ (IN)VISIBILITY, VULNERABILITY AND VIOLENCE \\ OF CHILDREN AND THE ELDERLY
}

MARIA GIULIA BeRNARDINI
Università di Ferrara

Fecha de recepción: 9-11-20

Fecha de aceptación: 17-3-21

Resumen: Dentro de la reflexión jurídica, el tema de la violencia tiene una relevancia crucial. En particular, la literatura crítica ha sacado a la luz el carácter estructural adoptado por ese fenómeno con respecto a determinados sujetos y grupos, indicados como "vulnerables", poniendo de relieve como la cuestión se relaciona, incluso antes que con el plano jurídico, con aquel sociocultural, puesto que es en ese plano donde la violencia recibe una primera legitimación. Asumiendo este cuadro teórico-conceptual como base, la Autora reflexiona sobre el nexo que une (in)visibilidad, vulnerabilidad y violencia con respecto a las personas menores y ancianas; es decir, en relación con el factor "edad".

Abstract: In the legal reflection, the issue of violence has a crucial relevance. In particular, critical literature has focused on the structural character of this phenomenon with respect to certain subjects and groups, indicated as "vulnerable", stressing how, in addition to the legal field, the issue concerns also to the sociocultural one. Indeed, violence receives in the socio-cultural dimension its first legitimation. Assuming this theoretical-conceptual framework as a basis, the Author reflects on the nexus among (in)visibility, vulnerability and violence with respect to minors and the elderly; that is, in relation to "age".

Palabras clave: edad, edadismo, invisibilidad, vulnerabilidad, violencia Keywords: age, ageism, invisibility, vulnerability, violence 


\section{DERECHO, VIOLENCIA, INVISIBILIDAD: UNA PERSPECTIVA CRÍTICA}

La relación entre violencia y derecho es notoriamente ambivalente, tal vez contradictoria. Por un lado, una de las funciones principales del derecho es la de evitar que se lleven a cabo actos violentos por parte de los asociados -entendidos, en primer lugar, aunque no de forma exclusiva, como un uso arbitrario de la fuerza. Por otro lado, su carácter autoritario ha llevado a algunos a considerar que la violencia pueda estimarse como parte del derecho mismo o, por lo menos, de algunas de sus manifestaciones como, por ejemplo, las técnicas (represivas) de resolución de los problemas sociales ${ }^{1}$. Así, algunos sectores del derecho (sobre todo el penal) serían violentos al producir consecuencias violentas en algunos sujetos y cuerpos ${ }^{2}$ y por el hecho de que, normalmente, se desinteresan de los efectos producidos. Desde un punto de vista critico $^{3}$ se hace necesario contrarrestar tales efectos.

De un modo más general, el tema de la violencia asume una relevancia crucial dentro del debate jurídico ya que éste "sigue estando impregnado de confusiones teóricas y prácticas, que nacen del solapamiento irresuelto entre los temas de la fuerza y de la violencia en su colocación en la esfera pública

1 * Este trabajo es la versión en español, revisada y actualizada, del ensayo M.G. BERNARDINI “(In)visibili? La vulnerabilità alla violenza di chi non ha l'età”, GenIUS - Rivista di studi giuridici sull'orientamento sessuale e l'identità di genere, vol VII, núm. 2, 2020, pp. 23-34. Agradezco a Anna De Giuli por la traducción.

Para un primer acercamiento a algunas de las cuestiones relevantes sobre esta temática, empezando por la profundización del pensamiento de Walter Benjamin, véase C. MENKE, "Law and Violence", Law E Literature, núm. 22, vol. 1, 2010, pp. 1-17; en una óptica filosófico-política, véase también V. FABBRIZI, “On law, power and violence: from Christoph Menke to Hannah Arendt. A critical analysis", Philosophy kitchen, núm. 4, 2017, pp. 33-42 y A. CATANIA, G. PRETEROSSI (eds.), Forme della violenza, violenza della forma, ESI, Napoli, 2007.

2 O. GIOLO, "Oltre la critica. Appunti per una contemporanea teoria femminista del diritto", Diritto \& questioni pubbliche, núm. 15, vol. 2, 2015, pp. 63-82, p. 76.

3 Este trabajo adopta una aproximación critica al derecho y, más específicamente, asume la óptica de las llamadas teorías críticas del derecho. Para una mayor profundización, se remite al clásico G. MINDA, Postmodern legal movements. Law and jurisprudence at century's end, New York University, New York, 1995. Véase también los más recientes B. BIX, Jurisprudence: theory and context, Sweet \& Maxwell, London, 1996 y E. CHRISTODOULIDIS, R. DUKES, M. GOLDONI (eds.), Research handbook on critical legal theory, Edward Elgar Publishing, Glasgow, 2019. También puede verse al respecto M.G. BERNARDINI, O. GIOLO (eds.), Le teorie critiche del diritto, Pacini, Pisa, 2017 y EAD. (eds.), "Le teorie critiche del diritto. Saperi, politica, società", Materiali per una storia della cultura giuridica, vol. XLIX, núm. 2, 2019, pp. 315-470.

DERECHOS Y LIBERTADES

ISSN: 1133-0937

Número 46, Época II, enero 2022, pp. 273-295

DOI: https://doi.org/10.20318/dyl.2022.6521 
y privada" ${ }^{4}$. Se trata, como es sabido, de ámbitos en los que solo una lectura anacrónica (o, podría aventurarse, ideológica ${ }^{5}$ ) permite leerlos en términos dicotómicos o de separación neta. En verdad, ya desde hace tiempo, más allá de haber identificado la naturaleza política de los límites entre lo público y lo privado ${ }^{6}$, la reflexión (filosófico-)jurídica -además, no solo aquella atribuible al conjunto de las teorías críticas- ha puesto de relieve la continuidad de las dos esferas para llamar la atención sobre la necesidad de reconocer la relevancia que tienen las relaciones de poder tanto dentro de la esfera pública como de aquella privada.

Desde esta óptica, se destacan las asimetrías de poder que existen entre los sujetos (por lo general, por relacionarse con grupos sociales específicos) y la violencia puede interpretarse como una de las modalidades del mismo ejercicio del poder, cuyo fin es el mantenimiento de una relación jerárquica entre sujetos ${ }^{7}$. Por lo tanto, destacan dos cuestiones fundamentales, interconectadas entre sí, sobre las especificidades de los sujetos y de los cuerpos involucrados en las dinámicas de poder, que se reflejan incluso en la visibilidad de este fenómeno: se trata, en otras palabras, de entender quién ocupa la posición de dominancia y quién la de subordinación.

A este respecto, es posible identificar algunas constantes: representando al Otro ${ }^{8}$, mujeres, menores de edad, personas con discapacidad, ancianos, personas de una etnia diferente de la occidental, no sedentarios, no cristianos

$4 \quad$ Ivi, p. 73. Traducción propia.

5 Este, por ejemplo, es el planteamiento neoliberal; entre las aportaciones doctrinales más recientes a este respecto, véase O. GIOLO, Il diritto neoliberale, Jovene, Napoli, 2020.

$6 \quad$ Véase por lo menos J. TRONTO, Moral boundaries: a political argument for an ethics of care, Routledge, New York, 1993.

7 Con las palabras de Hannah Arendt: “fenomenológicamente se acerca a la fuerza individual, ya que los instrumentos de la violencia, como todos los demás instrumentos, se crean y utilizan con el único fin de multiplicar la fuerza natural hasta el punto de que, en el último estadio de desarrollo, puedan ocupar su lugar" (traducción propia de H. ARENDT, On Violence, Harcourt Bace J., London, 1970, p. 46).

8 La literatura sobre la temática de la alteridad y sobre lo que puede considerarse una "eterna guerra de uno contra el otro" -parafraseando a G.A. STELLA, Stella, negri, froci, giudei E co.: l'eterna guerra contro l'altro, Rizzoli, Milano, 2009-, practicada utilizando la violencia en cada una de sus manifestaciones, es tan extensa que no es posible tratarla en este lugar. Al menos desde Aristóteles, el tema de la alteridad es central en el pensamiento filosófico; sin pretensiones de exhaustividad, me remito a: E. LEVINÁS, Totalità e infinito. Saggio sull'esteriorità (1961), Jaca Book, Milano, 2016; P. RICOEUR, Sé come un altro (1990), Jaca Book, Milano, 1993; F. JULLIEN, L'apparizione dell'altro. Lo scarto e l'incontro, Feltrinelli, Milano, 2020. Sobre la relación entre la alteridad y la violencia, véase: L. SAMONÀ (ed.), “Filosofia e violenza: dialettica,

ISSN: 1133-0937

DOI: https://doi.org/10.20318/dyl.2022.6521
DERECHOS Y LIBERTADES

Número 46, Época II, enero 2022, pp. 273-295 
o con identidades de género no reconducibles al paradigma heteronormativo $^{9}$, suelen ocupar más a menudo el segundo polo de la relación, sufriendo el ejercicio del poder por parte de quien se identifica en cada momento como sujeto "dominante". Y este es un poder que, no pocas veces, se ejercita de forma violenta, hasta el punto de que puede considerarse "violencia" cualquier forma de abuso de poder y control que se exteriorice en un abuso físico, sexual, psicológico o económico.

Por tanto, no es casualidad que la literatura crítica sobre la temática de la violencia sea ya abundante y que, desde hace tiempo, se haya destacado el carácter estructural de ese fenómeno con respecto a determinados sujetos y grupos (usualmente denominados "vulnerables") ${ }^{10}$, poniendo de relieve cómo la cuestión está relacionada con el plano socio-cultural, incluso antes que con el jurídico -o además del plano jurídico-, porque es en este lugar donde la violencia recibe su primera legitimación ${ }^{11}$.

Con el fin de reconocer los casos de violencia -que claramente constituyen una precondición para la identificación de herramientas eficaces de contraste-, la cuestionada distinción entre público y privado adopta una re-

conflitto, alterità", Giornale di Filosofia, núm. 32, vol. 2, 2010; G. STRUMMIELLO, "Violenza dell'umano/(Non-)violenza dell'inumano", Annali d'Italianistica, núm. 35, 2017, pp. 37-50.

9 Quizás merece la pena explicitar que el concepto de heteronormatividad se refiere a la existencia de un paradigma como fundamento de las normas morales, sociales y jurídicas basado en la premisa de que hay una orientación sexual correcta, la heterosexual, que existe coincidencia entre sexo biológico y género y que existe una natural y necesaria complementariedad entre hombre y mujer. Para una profundización sobre el tema, véase, por ejemplo, la sección monográfica E. ABBATECOLA, L. STAGI (eds.), “L'eteronormatività tra costruzione e riproduzione", About Gender, núm. 4, vol. 7, 2015.

10 En particular, cuando se ejercita sobre mujeres y personas menores de edad. Dentro de una bibliografía muy abundante, en el ámbito de la literatura italiana véase, entre otros, los recientes L. RE, E. RIGO, M. VIRGILIO (eds.), “Le violenze maschili contro le donne”, numero monográfico de Rivista Studi sulla questione criminale, 2019, vol. 1-2; B. PEZZINI, A. LORENZETTI (eds.), La violenza di genere dal Codice rosso al Codice Rocco. Un itinerario di riflessione plurale attraverso la complessità del fenomeno, Giappichelli, Torino, 2020.

11 Por ejemplo, las normas de género que transmiten un concepto estereotipado de masculinidad -cuando esta se asocia a la dominación masculina- favorecen la adopción de conductas violentas dentro del ámbito familiar, lo cual se traduce en la realización de actos violentos hacia la pareja y maltratos hacia personas menores de edad. A este respecto, véase, por ejemplo C. ODDONE, “'Tutti gli uomini lo fanno'. Il ruolo della violenza nella costruzione sociale della maschilità: il punto di vista dei maltrattanti", About Gender, núm. 6, vol. 11, 2017, pp. 74-97. El artículo se inserta dentro de una sección monográfica más amplia sobre la "construcción de lo masculino": S. CICCONE, K. NARDINI (eds.), "Maschilità: tras/formazione, cambiamenti, resilienze e riconfigurazioni”, About Gender, núm. 6, vol. 11, 2017.

DERECHOS Y LIBERTADES

ISSN: 1133-0937

Número 46, Época II, enero 2022, pp. 273-295

DOI: https://doi.org/10.20318/dyl.2022.6521 
levancia especial. La presencia de un espacio cerrado, apartado de la vista de las instituciones -que, a pesar de las críticas mencionadas al principio, mantienen un rol de tutela de los derechos-, favorece la aparición y la perpetuación de las dinámicas violentas y, ocultándolas, hace que resulte más difícil intervenir ${ }^{12}$. Por esa razón, la sinergia entre actores públicos y privados se convierte en un elemento fundamental para identificar los factores sistémicos de la violencia y contrarrestarlos.

Así pues, no es casualidad que ya desde hace un tiempo el estudio de las relaciones entre (in)visibilidad, vulnerabilidad y violencia haya adquirido una importancia creciente -aunque en su mayor parte siga siendo sectorialdentro de la reflexión (filosófico-)jurídica.

La significación de una temática que plantea relevantes cuestiones socioculturales además de jurídicas y la inadecuación tanto de los instrumentos normativos de prevención y contraste de los que disponemos en la actualidad, como de las mismas categorías conceptuales de las que aún hacemos uso para captar la vastedad y complejidad de estas interacciones, han llevado a que un número cada vez mayor de estudiosas y estudiosos pongan el foco en la urgencia de enfrentarse de manera sistemática a los desafíos presentados por ese objeto de investigación.

Desafortunadamente, esta temática se visibiliza según una lógica "emergencial", por la atrocidad de algunos casos de crónica o por darse a conocer en el plano público su extensa difusión, con ocasión de algunas "jornadas" dedicadas específicamente a ello. Por tanto, la sobreexposición (sobre todo mediática) y la invisibilidad se entrelazan sin llegar a abarcar del todo el carácter estructural integrado por el fenómeno de la violencia.

Así, esta invisibilidad, la cual se genera y se favorece por la ausencia de datos al respecto ${ }^{13}$ y por el hecho de que estos actos de violencia se llevan a

12 No obstante, no se excluye que la violencia pueda llevarse a cabo incluso en las "fronteras": piénsese en aquellos fenómenos como el trafficking y los procesos migratorios, en particular los que llevan a cabo los menores extranjeros no acompañados. Como confirmación de la invisibilidad de estos sujetos, como es fácil de imaginar, los datos sobre las formas de violencia padecidas por los menores en el proceso migratorio son incompletos; un intento de investigación es el de A. JUD, E. PFEIFFER, M. JARCZOK, “Epidemiology of violence against children in migration: a systematic literature review", Child Abuse E Neglect, núm. 108, 2020, pp. 1-13 (artículo 104634).

13 Se trata de datos que son difíciles de conseguir, justamente por la peculiaridad del tema y por la dificultad comprensible que tienen las víctimas a la hora de hacer "visible" su propia experiencia. Por lo menos en Italia, la mayoría de los datos disponibles son con respec- 
cabo dentro de espacios cerrados, conlleva también la invisibilidad de los sujetos hacia quienes se ejecutan estos actos de violencia.

En este sentido, la invisibilidad se puede interpretar como una forma aguda de discriminación ${ }^{14}$, que se caracteriza por una exclusión de tipo estructural y se alimenta de los estereotipos socialmente difundidos. Entre ellos, recordemos el "estigma" de la vulnerabilidad, mencionado anteriormente, en su acepción "clásica": eso es, como característica de un sujeto (el "sujeto vulnerable") que no se corresponde con la imagen del Sujeto ("fuerte", o sin ninguna otra connotación) por faltarle una o mas características que son propias de este último.

No es casualidad que, recientemente, el término "vulnerabilidad" haya sido objeto de una labor de resemantización para liberarlo de las connotaciones esencialistas, pasivizantes y estigmatizantes que a lo largo del tiempo lo han venido caracterizando ${ }^{15}$. Dichos rasgos parecen seguir estando difundi-

to a la violencia de género, pero no han faltado intentos de recopilación de datos en relación con otros sujetos. Por ejemplo, en junio 2020, el ISTAT (Instituto Nacional de Estadística italiano) ha seguido, en línea con los estudios previos, una investigación buscando profundizar en la temática de la violencia contra los menores y a los daños a niñas/os y adolescentes, aportando evidencias empíricas en tema de stalking con víctimas menores de edad y de delitos sexuales contra los menores de edad, con el fin de apoyar los trabajos de la Comisión parlamentaria para la infancia y la adolescencia (Istat, Indagine conoscitiva sulle forme di violenza tra $i$ minori e ai danni di bambini e adolescenti, giugno 2020, https://www.istat.it/it/files//2020/06/ Istat_Memoria-scritta_Violenza-tra-minori_1-giugno-2020.pdf). De la misma manera, el elevado número de muertos registrado dentro de las estructuras residenciales y semi-residenciales durante el lockdown ha puesto de manifiesto la necesidad de recabar un mayor número de datos con respecto a la condición de aquellos que están alojados, pidiendo hacer hincapié en aquellas cuestiones que, por lo general, han sido objeto de debate público solo de forma episódica, por haberse llevado a cabo dentro de esas estructuras actos de violencia como -entre otros- agresiones, inmovilizaciones o abandonos. Algunos datos se han recopilado gracias a la acción conjunta del Instituto Superior de Sanidad y del Garante Nacional de los derechos de las personas detenidas y privadas de la libertad personal, los cuales han confluido en el documento "Survey nazionale sul contagio COVID-19 nelle strutture residenziali e sociosanitarie", 5 mayo 2020.

14 H. HONNETH, A. MARGALIT, Recognition, Proceedings of the Aristotelian Society, 2001, Supplementary Volume 75, pp. 111-123.

15 Dicho cambio ha empezado a raíz del "vulnerability turn". A este respecto véase L. BURGORGUE-LARSEN, "La vulnérabilité saisie par la philosophie, le sociologie et le droit. De la nécessité d'un dialogue interdisciplinaire", en EAD. (eds.), La vulnérabilité saisie par les juges en Europe, Paris, Pedone, 2014, pp. 237 ss. El debate sobre este tema ha ido animándose; sin tener la pretensión de exhaustividad, dentro de la extensa literatura internacional véase M. FINEMAN, A. GREAR (eds.), Vulnerability. Reflections on a new ethical foundation for law and politics, Ashgate, Aldershot, 2013 y C. MACKENZIE, W. ROGERS, S. DODDS (eds.), 
dos en el debate jurídico al admitirse, e incluso requerirse, un enfoque paternalista y de protección (solo) hacia las personas vulnerables por parte de las instituciones que, de esa manera, se muestran responsive ${ }^{16}$. En particular, en los últimos años la reflexión crítica sobre la vulnerabilidad ha destacado los riesgos que lleva aparejado el uso de la presunción según la cual algunos sujetos (eso es, los que pertenecen a grupos históricamente discriminados, excluidos y/o oprimidos) son vulnerables y, por tanto, necesitan protección ${ }^{17}$. Entre esos riesgos, la falta o el reconocimiento parcial de la agency individual (y, por consiguiente, de los derechos de autodeterminación y autonomía) asume una importancia particular sobre todo cuando esta conlleva la falta de reconocimiento de la capacidad (jurídica y/o de obrar) del sujeto ${ }^{18}$.

Vulnerability. New Essays in Ethics and Feminist Philosophy, Oxford University Press, New York, 2014. Dentro de la bibliografía italiana, véase además TH. CASADEI (ed.), Diritti umani e soggetti vulnerabili. Violazioni, trasformazioni, aporie, Giappichelli, Torino, 2012 y, entre los trabajos más recientes, veáse B. PASTORE, O. GIOLO (eds.), Vulnerabilità. Analisi multidisciplinare di un concetto, Carocci, Roma, 2018; A. FURIA, S. ZULLO (eds.), La vulnerabilità come metodo, Carocci, Roma, 2020; G. ZANETTI, Filosofia della vulnerabilità. Percezione, discriminazione, diritto, Carocci, Roma, 2019 (traducido también en español como Filosofía de la vulnerabilidad. Percepción, Discriminación, Derecho, Editorial Dykinson, Madrid, 2020); R. FULCO, Soggettività e potere. Ontologia della vulnerabilità in Simone Weil, Quodlibet, Macerata, 2020; A. DE GIULI, "Sul concetto di 'vulnerabilità' secondo la Corte di Giustizia UE", Diritto penale e Uomo (DPU), núm. 10, 2020, pp. 1 ss.

16 Es emblemático, al respecto, el conocido texto: R.E. GOODIN, Protecting the vulnerable: a reanalysis of our social responsibilities, University of Chicago Press, Chicago, 1985. El concepto de "responsiveness" ha sido desarrollado en particular por Martha Fineman; además del conocido M. FINEMAN, "The vulnerable subject and the responsive State", Emory Law Journal, núm. 60, 2010, pp. 151-275, entre los trabajos más recientes, véase EAD., "Vulnerability and social justice", Valparaiso University Law Review, núm. 53 vol. 2, 2019, pp. 341-370.

17 Se trata, por otra parte, de riesgos que normalmente empeoran por el hecho de que, en relación con aquellos que se identifican como "sujetos vulnerables", a menudo se recurre a palabras como "vulnerabilidad", "debilidad" y "fragilidad" como si fueran sinónimos cuando no lo son: de hecho, a la vulnerabilidad le pertenece una dimensión relacional (se es vulnerable en relación con algo o alguien) que, en cambio parece estar ausente cuando se hace referencia a la debilidad y a la fragilidad.

18 En mi opinión, la tematización de la relación entre vulnerabilidad (ontológica) y capacidad constituye la línea de estudio más interesante del debate sobre el tema y, quizás, una de las pocas que parecen idóneas para producir consecuencias apreciables jurídicamente a partir del replanteamiento de la subjetividad jurídica y, más precisamente, de la noción de capacidad. Entre la bibliografía más reciente sobre el tema de la subjetividad jurídica (con atención particular al sujeto de derecho privado y al perfil de constitucionalización de la persona), véase por ejemplo F. BILOTTA, F. RAIMONDI (eds.), Il Soggetto di diritto. Storia ed evoluzione di un concetto nel diritto privato, Jovene, Napoli, 2020.

ISSN: 1133-0937

DOI: https://doi.org/10.20318/dyl.2022.6521
DERECHOS Y LIBERTADES

Número 46, Época II, enero 2022, pp. 273-295 
En efecto, la asignación de un sujeto al grupo de los “sujetos vulnerables" puede llegar a producir una distinción entre el Sujeto (no vulnerable), hacia quien el ordenamiento liberal tiene la obligación de adoptar una actitud anti-paternalista, y aquellos sujetos vulnerables, que parecen requerir la adopción de una orientación paternalista en razón de su vulnerabilidad intrínseca $\mathrm{y}$, en ese sentido, acaban siendo titulares de una subjetividad "débil" 19 . Además, la adopción de una concepción esencialista de vulnerabilidad lleva también a esconder la relación que hay entre vulnerabilidad y poder, "o, mejor dicho, entre vulnerabilidad y sometimiento, que hace que la vulnerabilidad esté intrínsecamente conectada con las relaciones de dominio y con la noción de violencia" ${ }^{20}$.

En el debate más reciente, el intento de superar los inconvenientes que nacen del uso de la noción de vulnerabilidad ha llevado a centrar la atención teórica y jurisprudencial, por un lado, en la condición de vulnerabilidad en la que se encuentran ciertos sujetos y, por el otro, en los procesos de creación de la vulnerabilidad (la denominada vulnerabilidad patógena) -los cuales se centran en la "vulneración" de los derechos fundamentales-, así como en los lugares que favorecen la instauración de las antes mencionadas dinámicas de subordinación encubiertas bajo el velo de la invisibilidad. Por tanto, es en esta perspectiva en la que reflexionaré sobre el nexo entre (in)visibilidad, vulnerabilidad y violencia, en la acepción específica que adquiere con respecto a la edad. Normalmente, los menores y las personas mayores de edad se consideran (grupos de) "sujetos vulnerables" ${ }^{21} \mathrm{y}$, a pesar de las dificultades a la hora de hacer sobresalir datos al respecto, la crónica da cuenta de numerosos casos de violencia que se perpetran hacia ellos. Antes de llevar a

19 Sobre el punto, también en relación con la edad, véase M.C. BARRANCO AVILÉS, "Human rights and vulnerability. Examples of sexism and ageism", The Age of Human Rights Journal, núm. 5, 2015, pp. 29-49, y asimismo M.C. BARRANCO AVILÉS, C. CHURRUCA MUGURUZA (eds.), Vulnerabilidad y protección de los derechos humanos, Tirant lo Blanch, Madrid, 2014.

20 Traducción propia de S. ZULLO, "Lo spazio sociale della vulnerabilità tra pretese di giustizia e pretese di diritto. Alcune considerazioni critiche", Politica del diritto, núm. 3, 2016, pp. 475-508, p. 479; F. PIZZOLATO, “Il governo politico del potere economico nella dimensione costituzionale", en Id. (ed.), Libertà e potere nei rapporti economici. Profili giuspubblicistici, Milano, Giuffrè, 2010, pp. 47-76 en particular pp. 61 ss.

21 Aunque no será objeto de análisis en este trabajo, es oportuno subrayar como, sobre todo como consecuencia de las crisis económicas que influyen de modo significativo sobre la precarización laboral, también los jóvenes serán considerados cada vez con mayor frecuencia "sujetos vulnerables". 
cabo algunas consideraciones sobre tales aspectos, puede ser útil reflexionar brevemente sobre la inestabilidad de la categoría "edad" y sobre cuál es la edad del sujeto-parámetro dominante.

\section{2. ¿NO TENGO (AÚN O YA) LA EDAD? EL SUJETO (SOCIALMENTE) DOMINANTE}

Desde la época antigua, la vida de los seres humanos se ha dividido de forma convencional en etapas ligadas a la edad: si en La Retórica Aristóteles identificaba tres (juventud, madurez y vejez) ${ }^{22}$, hacia el final del año seiscientos, con la desaparición de las grandes epidemias, el decremento de la tasa de mortalidad infantil favoreció la dilucidación de una fase adicional, la infancia, que con el paso de los siglos ha ido adquiriendo una mayor autonomía con respecto a la juventud. En efecto, ni siquiera el proceso de multiplicación y especificación de los estadios que preceden a la madurez (es decir, la edad adulta) parece poder circunscribirse únicamente a tales fases, como puede notarse claramente si solo se toma en consideración la adolescencia, es decir, el período de transición entre la infancia y la edad adulta que precede a la juventud ${ }^{23}$.

El mismo proceso se da con respecto al estadio de la edad cronológicamente sucesivo a la madurez. De hecho, el alargamiento progresivo de la vida media que se ha verificado en las últimas décadas, causa del aumento de la esperanza de vida y del conocido fenómeno del envejecimiento de la población mundial, ha llevado a identificar varios "tipos" de vejez, también integrables en un principio en una subdivisión por estadios ${ }^{24}$. No es casualidad que, cada vez de forma más frecuente, se haya hablado de la vejez no como una categoría unitaria y unificadora, sino como una "tercera" y una "cuarta" edad o, como alternativa, se adscriba a los individuos a una categoría de "jóvenes ancianos", de "ancianos" o de "grandes ancianos" 25.

$22 \quad$ ARISTÓTELES, Rhetorica, II, 12-14, 1388 b-1390 b.

23 Más ampliamente, véase en R. BODEI, Generazioni. Età della vita, età delle cose, Laterza, Roma-Bari, 2015.

24 Otro criterio clasificatorio puede ser aquel relativo a la autonomía. Piénsese en la diferencia entre healthy aging y "no-autosuficiencia".

25 A este respecto, es oportuno hacer algunas aclaraciones. La primera es de carácter clasificatorio: según las definiciones adoptadas a nivel internacional, en el mundo occidental la senectud empieza cuando se cumplen sesenta y cinco años. No obstante, últimamente se debate sobre la posibilidad de elevar este umbral, adoptando una concepción "dinámica" del proceso 
Esta tendencia definitoria y clasificatoria -casi obsesiva en su obra de multiplicación de los estadios (o de los estados) existenciales-, acaba por generar una confusión significativa con relación a la age identity del individuo, ya sea porque ésta no parece ser reducible al simple dato del Registro Civil (por ejemplo, referirse solamente a la edad de una persona no dice nada con respecto a su condición psicofísica), ya sea porque cambian continuamente las categorías de identidad, en las cuales cada uno puede decidir reconocerse y a las cuales con frecuencia se recurre para reconocerlo, influyendo en la visibilidad de su subjetividad. En las relaciones intersubjetivas, por otra parte, la edad constituye un criterio de juicio primario ${ }^{26}$, que viene empleado para categorizar a las personas y por norma alcanza una base justificativa de consideraciones ligadas a la capacidad de un sujeto y a su adecuación de cara a desempeñar un rol social determinado. Sin embargo, esta operación no es en absoluto sencilla por numerosas razones.

En primer lugar, no pudiéndose oponer a un proceso de envejecimiento que, a pesar de los progresos obtenidos en la técnica, presenta aún un carácter universal, las personas cambian su pertenencia categorial en relación con la edad de modo involuntario con el paso del tiempo, lo que hace que se vuelva una tarea aún más ardua determinar a qué categoría pueda ser adscrita la condición existencial de cada uno. Además, el umbral de paso entre los diferentes estadios es una variable fuertemente dependiente de la cultura y del contexto,

de envejecimiento. Como ejemplo, véase la propuesta de la Sociedad Italiana de Gerontología y Geriatría que, con ocasión del sexagésimo tercer Congreso Nacional, en noviembre de 2018, ha propuesto elevar el umbral a los 75 años. Una ulterior aclaración concierne al hecho de que el alargamiento de la expectativa de vida se ve afectada de modo significativo también por la variable geográfica: por ejemplo, si en 2017 la esperanza de vida en la Unión Europea era de 83,5 años para las mujeres y de 78,3 años para los hombres, en África era de 61 años para los hombres y 64 para las mujeres. Por lo tanto, cuando nos referimos al "envejecimiento rápido" de la población, por norma, nos referimos al contexto occidental. Por último, es necesaria una aclaración "lingüística": aún siendo consciente de que vejez y ancianidad no son sinónimos y que, al contrario, aquellos que han reflexionado específicamente sobre tales condiciones existenciales han rechazado explícitamente cada referencia a la ancianidad considerando a esta última un producto de la ideología neoliberal (a tal propósito, véase Simone de Beauvoir y Norberto Bobbio), por simplificar en este lugar haré uso de ambos términos, sin adherirme con ello al planteamiento neoliberal que ve la exaltación del (solo) sujeto anciano healthy y consumidor. El análisis de tal crítica está fuera del objeto del presente trabajo. Sobre el punto, véase más ampliamente en S. DE BEAUVOIR, La terza età (1970), Einaudi, Torino, 2002, y N. BOBBIO, De senectute e altri scritti autobiografici, Einaudi, Torino, 1996.

26 Véase A. MUCCHI FAINA, Troppo giovani, troppo vecchi. Il pregiudizio sull'età, Laterza, Roma-Bari, 2013, p. 4.

DERECHOS Y LIBERTADES

ISSN: 1133-0937

Número 46, Época II, enero 2022, pp. 273-295

DOI: https://doi.org/10.20318/dyl.2022.6521 
por tanto, la valoración de la edad de un individuo -en particular, si se dirige a certificar la juventud o la vejez y, así, a establecer la adecuación de su edadnormalmente depende de las finalidades perseguidas (a partir de aquellas laborales). Por lo tanto, no es infrecuente que las reflexiones relativas a la edad a menudo pongan de relieve el "no aún" y el "ya no", es decir, los "demasiado" que revelan la excedencia con respecto a la norma, la inadecuación de quién no tiene la edad justa y su falta de correspondencia con un parámetro de referencia que, ciertamente, se encuentra presente, aunque no de modo explícito y en el cual, en una óptica crítica, es posible identificar su propia antropología.

Entre sus connotaciones, para los fines que aquí nos interesan, es suficiente poner de relieve justo la edad: el sujeto-parámetro es el individuo adulto (varón), cuya personalidad está ya formada, la cual es, pues, más completa y socializada. Se trata de un sujeto racional, considerado en grado de conjugar energía y competencia, así como -factor decisivo con fines de reconocimiento de la plena subjetividad- de contribuir activamente al sistema productivo ${ }^{27}$. Tal capacidad le permite, además, alcanzar más fácilmente una posición apical dentro de la sociedad, de manera que se favorezca la instauración de una relación asimétrica entre quien presenta las características mencionadas y quien, careciendo de una o más de ellas, no se le considera en grado de satisfacer los requisitos demandados por el parámetro de referencia, por ejemplo, porque se entiende que no posee aún una personalidad completamente formada, o porque no está (aún o ya) en grado de cooperar con el bienestar social a través del desarrollo de una actividad productiva.

A nivel sociocultural, los efectos de este sistema de poder son múltiples: entre ellos, adquiere una relevancia particular el impacto producido por la "carencia" a veces relevante sobre la subjetividad de quien se encuentra en la condición de subordinación, a la cual he hecho referencia previamente hablando de "subjetividad debilitada", y que ha sido puesto de relieve eficazmente por la jurista estadounidense Catharine McKinnon cuando, en relación al carácter sexuado del sujeto-parámetro, se hacía la célebre pregunta: "are women human?" 28.

27 Esta, por ejemplo, es claramente la antropología asumida por John Rawls como base de su teoría de la justicia. Véase J. RAWLS, Una teoria della giustizia (1971), Feltrinelli, Milano, 2008.

28 C. MACKINNON, Are women human? And other international dialogues, Harvard University Press, Cambridge (MA), 2006. Para una profundización del pensamiento de MacKinnon, me remito a S. VANTIN, "Law as a weapon. Substantive equality in the legal and philosophical thought of Catharine MacKinnon", en R. DE ASÍS, F.J. ANSUÁTEGUI ROIG (eds.), Materiales de Filosofía del Derecho, núm. 1, 2019, pp. 1-17. 
La duda (claramente retórica) relativa a la plena humanidad de las mujeres $-\mathrm{y}$, extendiendo el pensamiento de McKinnon, de los varios sujetos no paradigmáticos- deriva del hecho de que la presencia del sujeto-parámetro lleva a reconocer los tratos distintivos de la "plena humanidad" únicamente en quien encarna sus características. Y es precisamente la presencia de tal sistema de poder lo que impide responder de modo completamente afirmativo a la pregunta referida por MacKinnon sobre la (plena) humanidad, articulada claramente en referencia a la edad.

La cuestión, en verdad, es compleja, a causa de la interacción de imágenes conflictivas ligadas tanto a la menor edad, como a la vejez; los dos trayectos, además, no viajan perfectamente en paralelo. En lo que se refiere a las personas menores ${ }^{29}$, sin llegar a los extremos del fenómeno de los denominados "feral children", basta pensar en como los estudios conducidos en el ámbito de las ciencias psicológicas y sociales han revelado que aún está difundida una imagen estereotipada de las niñas y de los niños, según la cual tales individuos carecerían de los atributos plenamente humanos que se adquieren con la socialización, es decir, el lenguaje, la sociabilidad y la inteligencia $^{30}$. Simétricamente, también los estereotipos relativos a la vejez socialmente más difundidos llevan aún a considerar que el deterioro creciente de las capacidades cognitivas y relacionales debidas a la senectud-capacidades consideradas fundamentales para el reconocimiento de la humanidad-incida inevitablemente sobre la lucidez de la persona anciana ${ }^{31}$, volviéndola manipulable y provoque con ello un regreso a la edad infantil, favoreciendo así la pérdida de los caracteres de la plena humanidad (de ese modo, entonces, el "aún no" relativo a la persona menor se sustituye por el "ya no" que caracteriza a aquella anciana) ${ }^{32}$.

29 Como en relación con la dialéctica ancianidad/vejez, por simplicidad expositiva, a continuación del trabajo haré (también) uso de la expresión "menores". Conviene observar que este sustantivo contiene ya en sí un reclamo a un parámetro de referencia, revelando así la falta de correspondencia y la inferioridad: ¿menor respecto a quién?

30 Véase S. LOUGHNAN, N. HASLAM, “Animals and androids: Implicit associations between social categories and nonhumans", Psychological Sciences, núm. 18, 2008, pp. 116-121.

31 No se trata de contestar el hecho de que la senectud pueda comportar el declive de alguna capacidad, sino el fundamento de las presunciones que implican un carácter de necesidad.

32 Véase A. MUCCHI FAINA, Troppo giovani, troppo vecchi. Il pregiudizio sull'età, cit., p. 17. Aún en los años setenta del siglo XX, Simone de Beauvoir se preguntaba de hecho "¿los viejos son seres humanos?" y respondía que "a juzgar por el modo en el que son tratados en nuestra sociedad es lícito dudar. Para nuestra sociedad, no tienen las mismas exigencias y los 


\section{LAS PERSONAS MENORES Y ANCIANAS: LA RELACIÓN CONTRO- VERTIDA CON EL PARÁMETRO JURÍDICO}

Ahora bien, esta falta (adecuada) de reconocimiento ha interesado también a la esfera jurídica, dentro de la cual, de modo no tan diferente de lo que sucede en el contexto social más amplio, el Sujeto paradigmático está notoriamente constituido por el hombre adulto.

Aunque la edad constituye uno de los elementos en relación con los cuales las fuentes normativas prevén con frecuencia una prohibición de discriminación ${ }^{33}$, en este contexto, no obstante, la visibilidad de las condiciones existenciales de las personas menores y de aquellas ancianas parecen sensiblemente diferentes.

En primer lugar, en el primer caso, el derecho establece explícitamente una edad de paso entre los estatus (el establecimiento de la mayoría de edad $)^{34}$. Por el contrario, no se pronuncia sobre el segundo: una vez alcanzada la adultez, la edad adquiere relevancia, en efecto, como requisito para

mismos derechos que los otros miembros de la colectividad: se les niega incluso el mínimo necesario. Los ancianos son deliberadamente condenados a la miseria, a los tugurios, a las enfermedades, a la desesperación" (Traducción propia de S. DE BEAUVOIR, La terza età, cit.). Si las palabras de la filósofa francesa parecían ya de persistente actualidad antes de la pandemia, la "masacre de los ancianos" avenida en los últimos meses dentro de las residencias socioasistenciales de todo el mundo no hace sino confirmar las condiciones de vida de tales individuos, que pasan necesariamente también por el reconocimiento de su plena subjetividad.

33 Se trata de una prohibición que no siempre es explícita. Por ejemplo, en el art. 3 de la Constitución italiana no es posible identificar una prohibición específica de discriminación por motivos de edad, que se tomará entonces en consideración mediante la cláusula "cualquier otra condición". Por otra parte, dentro de la Carta constitucional, la edad se pone más veces de relieve en referencia a las personas menores que a aquellas ancianas (para un primer acercamiento, véase P. PASSAGLIA, "I minori nel diritto costituzionale", Persona e danno, 2010, https://www. personaedanno.it/dA/bef1e1aada/allegato/AA_019215_resource1_orig.pdf). Al contrario, en la Carta de los derechos fundamentales de la Unión Europea existe una prohibición específica de discriminación en función de la edad (art. 21), y se toman en consideración tanto la subjetividad de las personas menores (art. 24) como la de las personas ancianas (art. 25).

34 Pero este "reconocimiento" no ha caracterizado siempre la esfera jurídica, donde el favor minoris es relativamente reciente, y se ha traducido en la presencia del derecho de los menores y de previsiones específicas que tienen como objeto la tutela de los menores. Por ejemplo, los Códigos liberales del s. XIX eran tendencialmente indiferentes con respecto a la exigencias materiales y psicológicas ligadas a la edad, probablemente por la necesidad de simplificación conectada con la teorización del sujeto único de derecho, ya desde hace un tiempo en fase de superación. Sobre el tema véase F. BILOTTA, F. RAIMONDI (eds.), Il Soggetto di diritto. Storia ed evoluzione di un concetto nel diritto privato, cit.

ISSN: 1133-0937

DOI: https://doi.org/10.20318/dyl.2022.6521
DERECHOS Y LIBERTADES

Número 46, Época II, enero 2022, pp. 273-295 
obtener determinados beneficios, aunque desde el punto de vista jurídico su progreso no comporta ningún cambio de estatus.

Además, también la evolución de los debates entorno a la representación de la subjetividad de las personas menores y ancianas, que repercuten sobre el reconocimiento explícito de su "derecho a tener derecho" (y por tanto sobre los tipos de derecho de los que son titulares reconocidos), no es la misma.

En lo que respecta a las personas menores, históricamente se ha asistido a un camino que ha llevado, al menos formalmente, a la superación de la idea de su no-subjetividad, que en realidad estaba difundida dentro de la esfera jurídica hasta hace un tiempo relativamente reciente. Lo demuestra, por ejemplo, el asunto de Mary Ellen Wilson que, a finales del siglo XIX, logró obtener la tutela de los abusos perpetrados por la familia de acogida únicamente porque un conocido activista de la época, Henry Bergh, contribuyó a dar a conocer su historia haciendo una apelación a la normativa vigente en aquel tiempo sobre la tutela de los derechos -no de los niños, aunque en aquellos años se comenzaron a adoptar las primeras legislaciones sobre el tema, sino- de los animales ${ }^{35}$. Aún hoy, por otra parte, la difusión de prácticas como aquella sobre la explotación y el trabajo infantil, así como sobre fenómenos como -entre otros- la trata de las personas menores, matrimonios precoces (denominadas "niñas esposas") o formas más o menos enmascaradas de "esclavitud" 36 , proponen nuevamente el tema de la falta de reconoci-

35 Entre las contribuciones que ligan la representación cultural de la infancia y de los derechos de los niños, en referencia a la bibliografía italiana y sin ningún propósito de exhaustividad se reenvía al menos a I. FANLO CORTES, Bambini e diritti. Una relazione problematica, Giappichelli, Torino, 2008; M. LALATTA COSTERBOSA, Il bambino come nemico. L'eccezione humboldtiana, DeriveApprodi, Roma, 2019; P. RONFANI, I diritti del minore. Cultura giuridica e rappresentazioni sociali, Guerini Scientifica, Milano, 1995, y EAD., "I diritti dei bambini: vecchie e nuove questioni", Sociologia del diritto, 2, 2013, pp. 107-130; TH. CASADEI, L. RE (eds.), "Children's Rights: tra violazioni e forme di tutela", Jura Gentium, 2015, https://www.juragentium.org/forum/infanzia/it/intro.html. Por último, para un excursus histórico e interdisciplinar que haga referencia específicamente a la violencia contra las y los menores, véase M. POLO, "Le violenze contro i minori: cause e conseguenze", Psychofenia, 2017, pp. 35-36, pp. 185-208. Más ampliamente, véase también I. FANLO CORTÉS, “'Viejos' y 'Nuevos' derechos del niño. Un enfoque teórico", Revista de Derecho Privado, núm. 20, 2011, pp. 105-126.

36 Aunque el foco sea más amplio respecto a aquel de la condición del/la menor, para una investigación interesante relativa a las formas de esclavitud antiguas y contemporáneas, interpretadas como procesos de humanización véase M. SIMONAZZI, TH. CASADEI (eds.), Nuove e antiche forme di schiavitù, Editoriale Scientifica, Napoli, 2018. Un foco específico sobre las personas menores víctimas de trata y explotación en Italia es el de Save the Children, en el informe de 2018 con el título Piccoli schiavi invisibili. Sobre los matrimonios forzados, dentro 
miento de la plena subjetividad de tales individuos, en particular cuando se analiza según una perspectiva de género.

Bien considerado, no obstante, en estos casos el problema se refiere principalmente al perfil de la efectividad de los derechos fundamentales y humanos, y no a aquel sobre su titularidad, que parece hoy gozar de un consenso difundido, al menos tras la entrada en vigor de la Convención ONU sobre los derechos de la Infancia y de la Adolescencia (CRC), última etapa internacional del proceso cultural que ha llevado al reconocimiento explícito de la titularidad de los derechos humanos para las y los menores y que ha favorecido la afirmación de una visión compleja de su subjetividad ${ }^{37}$. De hecho, dentro de la CRC se busca conjugar el reconocimiento de la capacidad ${ }^{38}$ de las personas menores (véase en particular, el derecho a ser oído en el art. $12^{39}$ ) con la debida protección especial por razón de la vulnerabilidad específica y, asimismo, de liberar a tales sujetos de la asociación de forma exclusiva al status filiationis ${ }^{40}$.

de una bibliografía en expansión, véase: E. DIÉZ PERALTA, El matrimonio infantil y forzado en el Derecho Internacional, Tirant lo Blanc, Valencia, 2019 y A. BONFANTI, "Matrimoni forzati, infantili e precoci e tutela dei diritti umani in Europa: considerazioni di diritto internazionale privato", GenIUS, vol VII, núm. 2, 2020, online first http://www.geniusreview.eu/2021/matrimoni-forzati-infantili-e-precoci-e-tutela-dei-diritti-umani-in-europa-considerazioni-di-diritto-internazionale-privato/; N. IGAREDA, "Forced marriage in Europe: from a migration problem in a global world to the old phenomenon of gender violence/Les mariages forcés en Europe: d'un problème lié à la migration dans un monde globalisé à l'ancien phénomène de la violence de genre", Rivista di Criminologia, Vittimologia e Sicurezza, núm. 9, vol. 2, 2017, pp. 4-12.

37 Por ejemplo, en el preámbulo de la Convención de la Haya del 20 de mayo de 1993 en materia de adopción internacional, la referencia es a las personas menores como personas y sujetos de derecho, capaces de tomar autónomamente sus propias decisiones.

38 Piénsese en la valoración de la capacidad de discernimiento y la capacidad de negociación de las personas menores, como parte de un proceso más amplio dirigido a reconocer a tales sujetos un rol activo en el caso en el que se deba tomar una decisión que les afecta.

39 Por regla general, el derecho a ser oído se entiende como una manifestación del derecho a la libertad de expresión y de aquel de agency. Se trata de una previsión que asume una importancia fundamental allá donde, por su trámite, a quien es menor, se le reconoce explícitamente como sujeto de derecho y actor social y, por tanto, titular del "derecho a los derechos".

40 En relación con los perfiles arriba mencionados, véase en particular arts. 31-40 CRC; la violencia se define en el art.19 CRC. En referencia específicamente al ordenamiento italiano, se ha observado que solo con la entrada en vigor del d. lgs. 154/2013 ha habido lugar para la reconceptualización teórica de la subjetividad jurídica de las personas menores, mediante la desvinculación de su calificación exclusiva como miembro del status filiationis (S. VANTIN, "Ordine sociale, gerarchie familiari e soggetti di diritto nella società globale. I diritti dei minori e la sfida del pluralismo", Jura Gentium, 2015, https://www.juragentium.org/forum/infanzia/it/ vantin.html). Más ampliamente, véase el análisis de L. FADIGA, “Da figlio a bambino: il fan-

ISSN: 1133-0937

DOI: https://doi.org/10.20318/dyl.2022.6521
DERECHOS Y LIBERTADES

Número 46, Época II, enero 2022, pp. 273-295 
Al contrario, el debate relativo al reconocimiento de la subjetividad de las personas ancianas aparece mayormente "polarizado", tanto que incluso el diálogo relativo a la oportunidad de que las Naciones Unidas adopten una convención específica para la tutela de sus derechos parece haberse estanca$\mathrm{do}^{41}$, principalmente a causa de la dificultad de identificar los "límites" del grupo en cuestión y de reconocer dentro del mismo alguna homogeneidad.

En particular, impide alcanzar el consenso necesario para la elaboración de una convención dedicada específicamente al reconocimiento de la incapacidad de los derechos humanos de considerar de un modo unitario (es decir, precisamente, como haciendo parte de un único grupo) a aquellos que están interesados en un proceso de "envejecimiento activo" y aquellos quienes, al contrario, se encuentran en una condición de no-autosuficiencia.

Mientras que en el primer caso la finalidad del derecho y de las instituciones es promover al máximo la autonomía de los individuos ${ }^{42}$, en el segundo se encuentran mayores resistencias culturales en relación con el reconocimiento de los derechos sobre un plano de igualdad, sobre todo en lo que concierne a los derechos de autonomía y -otra vez- la presunción relativa a la capacidad de los individuos.

Por otra parte, lo anterior es aún más cierto cuando la subjetividad de aquellos cuyos derechos se discute es aquella de las personas que son huéspedes de estructuras de long term care $^{43}$. A ese respecto, resulta oportuno señalar que, tam-

ciullo come persona titolare di diritti", Jura Gentium, 2015, https://www.juragentium.org/forum/ infanzia/it/fadiga.html.

Sin embargo, no estoy segura de que, en relación con el tema específico de la violencia, la desvinculación del status filiationis pueda darse por terminada: de hecho, bastante a menudo, la referencia se hace a las mujeres y a los/las menores, lo que sugiere -parece- considerar la relación entre madres e hijos (los unos y los otros, víctimas de violencia), más que las subjetividades distintas.

41 De modo diferente, desde el 2015 está en vigor la Convención interamericana sobre los derechos de las personas ancianas. Para una introducción véase F. SEATZU, "Sulla convenzione dell'organizzazione degli stati americani sui diritti delle persone anziane", Anuario Español de derecho internacional, núm. 31, 2015, pp. 349-366.

42 Dicha valoración se considera funcional también de cara a la prevención de los abusos y los maltratos. Entre los documentos más relevantes, elaborados principalmente por la plataforma AGE, véase la EU Charter of rights and responsibilities of older people in need of long-term care and assistance, https://age-platform.eu/publications/ eu-charter-rights-and-responsibilities-older-people-need-long-term-care-and-assistance.

43 Sobre este punto, señalamos el hecho de que una obra importante de monitorización y de tutela de los derechos fundamentales en Italia viene realizada por el Garante nacional de los derechos de las personas detenidas y privadas de la libertad personal, con el fin de 
bién a continuación de la aprobación de la Convención Onu sobre los derechos de las personas con discapacidad (CRPD), está en curso un proceso cultural general de revalorización de la capacidad en un sentido universal, receptor también de una atenta jurisprudencia, que está llevando al reconocimiento de la subjetividad y de los derechos de autonomía de los individuos considerados por regla general más "vulnerables" ${ }^{44}$ en una óptica convergente con aquella expresada por Stefano Rodotà a través del recurso de "autonomía supervisada" 45.

Además, se advierten novedades significativas también dentro de las narrativas, aun difundidas dentro de la esfera jurídica, que hacen referencia a las personas menores y ancianas considerándolas "sujetos vulnerables" y a las que el ordenamiento ha intentado proteger con razón de su condición particular ${ }^{46}$. De hecho, también en este contexto se puede identificar una tendencia creciente al liberar el término "vulnerabilidad" de una concepción "pasivizante" de la subjetividad allá donde cada vez con más frecuencia, más que (o, a veces, además de) a la vulnerabilidad intrínseca, se hace referencia a la condición de vulnerabilidad especial o particular ${ }^{47}$. En esta óptica, la aten-

evitar que tales estructuras puedan configurarse como modernas "instituciones totales" y que venga respetada su libertad de elección. Parece, pues, necesario reflexionar de un modo más profundo sobre la "condición libre" de tales sujetos, así como sobre los requisitos formales y sustanciales sobre los que estos forman su voluntad, y sobre el rol relativo llevado a cabo por el derecho. Sobre tales aspectos, véase por lo menos el reciente A. FACCHI, O. GIOLO, Libera scelta e libera condizione. Un punto di vista femminista su libertà e diritto, Il Mulino, Bologna, 2020.

44 De hecho, dentro de la CRPD el art. 12 reconoce la capacidad (jurídica y de obrar) universal y el art.19 el derecho a la vida autónoma e independiente. Puesto que le he dedicado un amplio espacio a la reflexión sobre este tema en otro lugar, sobre todo en referencia a la discapacidad, me permito remitir también a M.G. BERNARDINI, La capacità vulnerabile, Jovene, Napoli, 2021. Por lo que respecta al ordenamiento italiano, una pronunciación significativa a nivel jurisprudencial que va en el sentido de la revaloración de la subjetividad capaz (de la persona anciana) es la ordinanza n. 4709/2018 de la Corte di Cassazione; para un comentario véase M. DI MASI, “Ads, 'gap' cognitivi e senilità: la Cassazione presidia l'autodeterminazione degli autosufficienti", Rivista critica del diritto privato, núm. 2, 2018, pp. 307-319.

45 S. RODOTÀ, La vita e le regole. Tra diritto e non diritto, Feltrinelli, Milano, 2006, p. 22.

46 Recurriendo justamente al paradigma de la vulnerabilidad, Jonathan Herring desafía a la concepción dominante, en base a la cual las niñas y los niños no pueden tener los mismos derechos que los adultos por razón de su "vulnerabilidad especial", poniendo en discusión la presunción de competencia relativa a los adultos. Véase J. HERRING, Vulnerability, childhood and the law, Springer, Dordrecht, 2018.

47 Por ejemplo, en el caso de las personas menores, en la ley italiana 47/2017 se procede a la regulación en materia de medidas de protección de los individuos menores extranjeros no acompañados, motivando el cuidado específico en el art. 1, apartado 2 “a razón de su condición de

ISSN: 1133-0937

DOI: https://doi.org/10.20318/dyl.2022.6521
DERECHOS Y LIBERTADES

Número 46, Época II, enero 2022, pp. 273-295 
ción se pone sobre los procesos exógenos de creación de la vulnerabilidad (que se traducen también en la violación de los derechos fundamentales), así como sobre los lugares en los cuales se verifican tales acontecimientos ${ }^{48}$. Puesto que eso se observa también analizando los debates y los documentos normativos en los cuales se trata sobre el tema específico de la violencia, esta última puede, entonces, ser considerada como una forma de vulnerabilidad patógena y, como tal, justificar algunas consideraciones sobre la articulación del nexo existente tras esta última, la (in)visibilidad de las personas menores y ancianas y su condición de vulnerabilidad.

\section{VIOLENCIA Y VULNERABILIDAD EN RELACIÓN CON LA EDAD: MUCHOS "VACÍOS" Y POCOS “LLENOS"}

La emergencia de la pandemia, sobre todo en los primeros meses de lockdown, ha llamado lamentablemente la atención pública sobre un tema en realidad ya conocido que, no obstante, se ha agravado en los meses que acaban de transcurrir: se trata del "vacío" relativo a la ausencia de una adecuada concienciación de la relación entre violencia, invisibilidad y una edad que, a causa de su "demasiado", no puede ser considerada como paradigmática y, como tal, se liga a la vulnerabilidad ${ }^{49}$. El fenómeno es claramente complejo

mayor vulnerabilidad". En este caso, el riesgo de la categorización parece remoto, porque la vulnerabilidad se identifica considerando un factor relacional, es decir, la falta de acompañamiento. Nótese, además, que en el plano internacional el Comité de los derechos del niño ha abandonado la referencia a "grupos vulnerables de niños" en favor de la expresión "niños en situaciones vulnerables", justamente para hacer énfasis en la situación relacional en la cual se encuentra el menor. Para un examen más articulado y la referencia a los documentos relevantes, véase F. IPPOLITO, "La vulnerabilità quale principio emergente nel diritto internazionale dei diritti umani?", Ars interpretandi, núm. 2, 2019, pp. 63-93. Más ampliamente, y también en relación con los demás "grupos vulnerables", véase F. IPPOLITO, S. IGLESIAS SÃNCHEZ, Protecting Vulnerable Groups. The European Human Rights Framework, Hart Publishing Oxford, 2015. Por último, también sobre los "grupos vulnerables", véase F. MACIOCE, La vulnerabilità di gruppo, Giappichelli, Torino, 2021.

48 Con un carácter más específico sobre la tutela procesal penal (así como sobre la inadecuación del sistema procesal italiano en relación con las personas menores), con respecto al ordenamiento italiano se centra en tal cambio de perspectiva F. TRAPELLA, "La tutela del vulnerabile. Regole europee, prassi devianti, possibili rimedi", Archivio Penale, núm. 3, 2019, pp. 1 ss.

49 Aunque no pone una atención específica en la violencia, profundiza en algunas cuestiones relevantes que están relacionadas con las condiciones de vulnerabilidad y pandemia $\mathrm{V}$. LORUBBIO, "La considerazione delle persone vulnerabili tra forti proclami internazionali e deboli risposte interne, durante la prima fase di vigenza delle misure di contrasto al Covid-19", 
por las diferentes formas en las cuales puede exteriorizarse ${ }^{50}$, la pluralidad de los sujetos que pueden realizar las (citadas) conductas relativas (activas u omisivas) ${ }^{51}$, así como debido a los diferentes lugares (físicos o virtuales) en dónde éstas se concretan.

Claramente, el "vacío" se refiere, en primer lugar, a la ausencia de datos: se sabe, en realidad de un modo impreciso y con estimaciones que seguramente pecan por defecto a causa de la dificultad de efectuar mediciones oficiales del fenómeno, que han aumentado los casos de violencia (física y online) y que la invisibilidad de tales acontecimientos se ha visto favorecida por el confinamiento generalizado. El "vacío", asimismo, se refiere también a la tutela ofrecida por el ordenamiento: a la ya dificultad estructural de prevenir y contrastar el fenómeno objetivo (derivado principalmente de las razones culturales que plantean un problema de conocimiento y de concienciación incluso entre los propios sujetos que participan en las dinámicas violentas y tras los operadores) bien por la dificultad relativa a la aparición de lo "sumergido" y a la posibilidad de "incluir en la red" a los operadores ${ }^{52}$, bien por la infrafinanciación estructural del sector, se han añadido de hecho otras problemáticas. Entre ellas, la interrupción de los servicios de prevención y lucha contra la violencia que se ha registrado de un modo prácticamente generalizado durante la pandemia ha exacerbado ciertamente la condición de vulnerabilidad, tanto de las personas menores como de las personas ancianas ${ }^{53}$.

DPCE online, núm. 2, 2020, pp. 2615-2641; con respecto a las personas mayores, véase: F.H. LLANO ALONSO, "Ancianidad y vulnerabilidad en tiempos de pandemia - I e II", Scienza e pace Magazine, http://magazine.cisp.unipi.it/ancianidad-y-vulnerabilidad-en-tiempos-de-pandemia-2/ (primera parte) y http://magazine.cisp.unipi.it/ancianidad-y-vulnerabilidad-en-tiempos-de-pandemia-seconda-parte/ (segunda parte).

50 Piénsese, entre otras, en la violencia psicológica, sexual, física, económica o el abandono. Si bien no me parece que estas formas de violencia afecten únicamente a las personas menores o a aquellas ancianas, otras parecen cometerse principalmente contra los/las menores, como la violencia doméstica presenciada por éstos.

51 Se trata, como se conoce, de familiares, care givers profesionales o no profesionales y, sobre todo en el caso de las personas menores, de "pares" (también en el caso de la violencia "entre pares" la variable "género" tiene una relevancia significativa, ya que las modalidades en las cuales se ejerce la violencia son diferentes; prevalece, en particular, aquella psicológica).

52 A modo de ejemplo, basta pensar que por razones de privacidad los sistemas informativos de la sanidad, de las fuerzas del orden y de los servicios sociales no están conectados, por lo que se hace aún más difícil identificar los casos de violencia.

53 En lo referente a las personas menores, la atención a la violencia ha sido específica: véase el documento de UNICEF, Protecting children from violence in the time of COVID-19, agosto 2020. En relación con las personas ancianas, el tema de la violencia ha sido, en cambio,

ISSN: 1133-0937

DOI: https://doi.org/10.20318/dyl.2022.6521
DERECHOS Y LIBERTADES

Número 46, Época II, enero 2022, pp. 273-295 
Al respecto, cabe señalar como -al menos hasta la pandemia- la invisibilidad no parece haber afectado del mismo modo a las condiciones existenciales de las personas menores y de aquellas ancianas.

En relación a las primeras, aunque falta aún mucho por hacer, sobre todo en lo referente al aspecto de la prevención, se viene reconociendo la importante obra de asociacionismo del sector -y su impulso "desde abajo", así como de las instituciones y de los organismos internacionales y nacionales $^{54}$ - en relación a la recogida de los datos, a la difusión de la concienciación sobre la existencia del fenómeno y a la identificación de los instrumentos (también normativos) que permiten prevenir además de (o antes de) contrastar las múltiples formas de violencia ${ }^{55}$. Es oportuno señalar como, en tales casos, se ha ido poniendo cada vez más una atención especial al género: de hecho, la intersección entre género y minoría de edad expone a un riesgo mayor de sufrir una violencia de naturaleza sexual ${ }^{56}$ y amplifica asimismo,

puesto de relieve dentro de un contexto más amplio, encaminado a poner el foco sobre la titularidad que pertenece a estos sujetos de derechos humanos, así como a remarcar la presencia persistente de la discriminación estructural conocida como "edadismo"; véase, al menos el Policy Brief de las Naciones Unidas The impact of Covid-19 on older people, mayo 2020.

54 Ex multis, véase al menos UN Committee on the Rights of the Child, General Comment $\mathrm{n} .13$, The right of the child to freedom from all forms of violence, 18 abril 2011; en el contexto italiano, Autoridad garante de la infancia y la adolescencia, La Convenzione delle Nazioni Unite sui diritti dell'infanzia e dell'adolescenza: conquiste e prospettive a trent'anni dall'adozione, Roma 2019 (sobre la violencia, véase en particular la contribución de A. LEANDRO, C. ZONILE, La tutela da ogni forma di violenza, pp. 258-272).

55 Nótese como, como consecuencia de los escándalos mediáticos acontecidos recientemente, en el ordenamiento italiano se ha difundido aún más la concienciación relativa a la necesidad de intervenir sobre el sistema de la defensa de los derechos de las personas menores, con el fin de aumentar el grado de tutela. A este proceso se adscribe asimismo el debate relativo a la reforma del art. 403 c.c. en materia de intervención de la autoridad pública en favor de los menores, objeto de una (controvertida) propuesta de ley que data del año 2017 (atto Camera 4299), recientemente pretendida -como consecuencia del denominado "caso Bibbiano" - también de la Autoridad Nacional Garante de la infancia y la adolescencia. El "caso Bibbiano", muy conocido en Italia, hace referencia a la supuesta presencia de una red ilícita en la gestión del acogimiento familiar de las personas menores, que en el momento actual aún no ha concluido con ninguna apreciación de responsabilidad.

56 Al contrario, la pertenencia de género no parece incidir de modo significativo sobre la probabilidad de ser víctima de violencia "en general": si los niños tienen un poco más de riesgo de sufrir castigos corporales, la disparidad de género penaliza, en cambio, fuertemente a las niñas en el caso de violencia sexual. Para una profundización de la situación italiana y global, véase al menos el dossier TERRE DES HOMMES (ed.), La condizione delle bambine e delle ragazze nel mondo 2019, asimismo E. BERNACCHI, A. FABRIS, M. ZELANO, Studio multi-paese sui drivers della violenza all'infanzia. Rapporto Italia, Firenze, Istituto degli Innocenti, 2016.

DERECHOS Y LIBERTADES

ISSN: 1133-0937

Número 46, Época II, enero 2022, pp. 273-295

DOI: https://doi.org/10.20318/dyl.2022.6521 
de un modo significativo, la magnitud de la opresión, de la exclusión y de la discriminación sufridas ${ }^{57}$. A la luz de cuanto se ha observado, a pesar de que en la práctica el fenómeno de la violencia sobre y entre las personas menores constituye lamentablemente aún una práctica sumergida, no parece entonces que se pueda hablar de una completa invisibilidad del fenómeno.

Sin embargo no parece que lo mismo se pueda decir en relación con las personas ancianas, para las cuales la pandemia ha funcionado de un modo aún más significativo como catalizador de la atención pública, principalmente a causa del escándalo de las residencias sanitarias asistenciales que ha llevado a la "matanza silenciosa" de una generación entera ${ }^{58}$. Aunque se trata de un fenómeno todo lo contrario a nuevo, la violencia contra estos individuos se ha convertido en objeto de un análisis riguroso solo en tiempos muy recientes, principalmente en el ámbito de los estudios relativos a la salud pública y aquellos con relevancia penal; se considera, en efecto, que los estudios sobre el tema llevan un retraso de por lo menos veinte años con respecto a aquellos que tienen como objeto la violencia sobre las mujeres y los menores ${ }^{59}$.

Así, se comprende mejor como la invisibilidad tendencial del tema se acompaña de la concienciación relativa al hecho de que se trata de una cuestión que pone importantes desafíos en tema de tutela de los derechos humanos,

57 Como es conocido, esto sucede allá donde los sujetos contra quienes se realizan actos violentos o discriminatorios son portadores de múltiples identidades discriminadas y oprimidas (la llamada "interseccionalidad"). La reflexión en torno a la interseccionalidad, a la que dio comienzo Kimberlé Crenshaw con referencia a las mujeres de color, se ha ido ampliando hasta tener en cuenta la interacción de diferentes factores de discriminación. De Crenshaw, me remito al conocido K. CRENSHAW, "Mapping the margins: intersectionality, identity politics, and violence against women of color", Standford Law Review, vol. 43, núm. 6, 1991, pp. 1241-1299. Entre los trabajos más recientes, veáse por lo menos B. BELLO, Intersezionalità. Teorie e pratiche tra diritto e società, Milano, Franco Angeli, 2020 y D. MORONDO, C. DE LA CRUZ, E. LA SPINA (eds.), Desigualdades complejas e Interseccionalidad. Una revisión crítica, Editorial Dykinson, Madrid, 2020. Permítaseme reenviar también a M.G. BERNARDINI (ed.), Migranti con disabilità e vulnerabilità. Rappresentazioni, politiche, diritti, Jovene, Napoli, 2019.

58 Sobre todo con referencia a la situación en Italia, se concentran sobre esta temática diferentes contribuciones presentes en: M.G. BERNARDINI, S. CARNOVALI (eds.), Diritti umani in emergenza: dialoghi sulla disabilità ai tempi del Covid-19, IF Press, Roma, 2021.

59 Para una profundización sobre estos aspectos, véase J.E. STOREY, "Risk factors for elder abuse and neglect: A review of the literature", Aggression and violent behavior, vol. 50, 2020, pp. 1-13, y M.C. MAZZOTTI, S. PELOTTI, "Invecchiare liberi da violenza: il problema dell'abuso sulla persona anziana in ámbito sanitario”, Notizie di Politeia, vol. 36, 2019, pp. 90-97.

ISSN: 1133-0937

DOI: https://doi.org/10.20318/dyl.2022.6521
DERECHOS Y LIBERTADES

Número 46, Época II, enero 2022, pp. 273-295 
también debido al proceso de envejecimiento global ${ }^{60}$. Piénsese, por ejemplo, que las estimaciones oficiales más recientes a las cuales hace referencia la literatura relativa al fenómeno son principalmente aquellas publicadas en el 2011 por la OMS, quien prácticamente ha proporcionado la única definición, poniendo el acento -más allá de las formas más conocidas de violencia- también sobre la negligencia, sobre el abandono y sobre las formas graves de pérdida de dignidad y de respeto a menudo experimentadas por personas ancianas ${ }^{61}$.

Estas violaciones se verifican, en particular, dentro del contexto familiar y de las estructuras residenciales y semi-residenciales (las estructuras de long term care), que no por casualidad han sido definidas recientemente como "manicomios ocultos" ${ }^{62}$, para llamar la atención sobre el hecho de que las prácticas que se realizaban dentro de las instituciones de los manicomios y que, sobre todo en el período "basagliano" ${ }^{63}$ en Italia, suscitaron tanto escándalo por ser abiertamente lesivas de los derechos humanos -primera entre todas, la contención ${ }^{64}$ - continúan siendo practicadas a día de hoy. Como se puede intuir fácilmente, se trata de violaciones de derechos que se acentúan de modo significativo con una connotación de género, a causa de la interacción entre el edadismo y el sexismo, lo que provoca que la violencia que tiene lugar contra las mujeres ancianas sea aún más fácilmente invisible debido a la hostilidad cultural de considerarlas como "sujetos" y de un modo particular como "sujetos sexuados" 65 .

\footnotetext{
60 Véase WHO, World report on ageing and health, Geneva, 2015.

61 Sobre este punto, y para una profundización adicional, véase https://apps.who.int/ violence-info/elder-abuse/

62 G. MERLO, C. TARANTINO (eds.), La segregazione delle persone con disabilità: i manicomi nascosti in Italia, Maggioli, Santarcangelo di Romagna, 2018. La referencia exclusiva a la discapacidad contenida en el título no debe engañar: al cumplir los 65 anos de edad, las personas con discapacidad "desaparecen", para "convertirse" en personas ancianas no autosuficientes.

63 De Basaglia es considerado el fundador del concepto moderno de salud mental e iniciador de una aproximación psiquiátrica centrada en el reconocimiento de la dignidad de la persona con discapacidad mental y sobre su centralidad. Véase F. BASAGLIA, L'istituzione negata, Einaudi, Torino, 1968.

64 Para una introducción al problema, véase S. ROSSI (ed.), Il nodo della contenzione. Diritto, psichiatria e dignità della persona, Alpha \& beta Verlag, Merano, 2015; S. ROSSI, P. TINCANI (eds.), I diritti forzati. Conversazioni sulla follia a quarant'anni dalla legge Basaglia, Ornitorinco, Milano, 2018; V. MARZOCCO, "La disabilità mentale tra vulnerabilità soggettiva e controllo sociale. Il caso della sofferenza psichica", Rivista di filosofía del diritto, vol. VII, núm. 2, 2018, pp. 321-334.

65 En relación con las estructuras residenciales y semiresidenciales, se plantea también un elemento cuantitativo: las mujeres ancianas constituyen cerca del 75\% de las personas alo-
} 
Una vez más, entonces, emergen diversos "vacíos" que contribuyen a la invisibilidad de tales fenómenos: estos atañen a la concienciación sociocultural de su existencia, así como al plano de la tutela, que se externaliza principalmente en la incapacidad de actuar sobre el plano de la prevención ${ }^{66}$. Y es igualmente fundamental con el fin de garantizar una tutela efectiva, que se inviertan recursos significativos en el plano de una formación que no se dirija únicamente a sensibilizar en relación a las varias formas de violencia que pueden perpetuarse (elemento por cierto fundamental desde la óptica de la prevención y de la lucha), sino también (e incluso antes) a ver a tales personas, reconociéndolas como titulares de los derechos fundamentales y humanos $\mathrm{y}$, por tanto, también del derecho a ser libres de cualquier forma de violencia, tanto en el plano formal, como -y sobre todo- en aquel de la efectividad.

\author{
Maria GiUlia Bernardini \\ Departamento de Derecho \\ Università di Ferrara \\ Corso Ercole I d'Este, 37 \\ 44121 Ferrara, Italia \\ e-mail:mariagiulia.bernardini@unife.it
}

jadas. Las contribuciones científicas sobre el tema de la violencia contra las mujeres ancianas son ciertamente escasas, señalo dos volúmenes editados por H. BOWS, Violence against older women, Palgrave Macmillan, Cham (Sw), 2019.

66 En esta óptica, por cuanto concierne al ordenamiento italiano es, por tanto, no solo oportuna, sino ciertamente indispensable la acción de monitorización puesta en marcha por la Autoridad garante de los derechos de las personas detenidas o privadas de la libertad personal en referencia a las estructuras socio-sanitarias. Puede asimismo acogerse de manera positiva el hecho de que actualmente se encuentre a examen del Parlamento Italiano una propuesta de ley que busca prevenir y luchar contra las conductas de maltrato y de abuso en perjuicio de las personas menores y ancianas dentro de las estructuras públicas y privadas (véase la propuesta de ley Misure per prevenire e contrastare condotte di maltrattamento o di abuso, anche di natura psicologica, in danno dei minori nei servizi educativi per l'infanzia e nelle scuole dell'infanzia e delle persone ospitate nelle strutture socio-sanitarie e socio-assistenziali per anziani e persone con disabilità e delega al Governo in materia di formazione del personale. La referencia tanto al sector público como al privado parece, más que nunca, oportuna, ya que sugiere una continuidad entre los dos ámbitos. 